\title{
An Analysis of Relationship Between RAS Mutations and Prognosis of Primary Tumour Resection for Metastatic Colorectal Cancer Patients
}

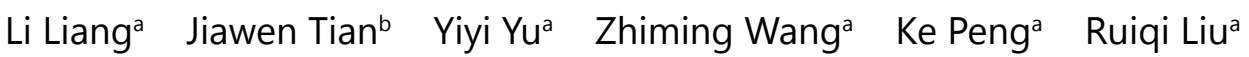 \\ Yan Wang ${ }^{\mathrm{a}}$ Xiaojing Xu $\mathrm{X}^{\mathrm{a}}$ Hong $\mathrm{Li}^{\mathrm{a}}$ Rongyuan Zhuang ${ }^{\mathrm{a}}$ Yuehong Cui ${ }^{\mathrm{a}}$ \\ Chouwen Zhu ${ }^{c}$ Tianshu Liua,c
}

aDepartment of Medical Oncology, Zhongshan Hospital, Fudan University, Shanghai, bepartment of Internal Medicine, Huadong Hospital, Fudan University, Shanghai, 'Evidence Based Medicine Center, Fudan University, Shanghai, China

\section{Key Words}

Colorectal Cancer • Asymptomatic • Metastatic • Unresectable • Primary Tumour Resection • RAS Gene

\begin{abstract}
Background/Aims: Non-radical primary tumour resection (PTR) of asymptomatic metastatic colorectal cancer (mCRC) can prolong survival time of some patients. Patients with mutated RAS gene have worse survival outcome. This study aimed to investigate the impact of RAS gene mutations on the prognosis of asymptomatic unresectable mCRC patients who underwent PTR. Methods: A retrospective observational cohort study was deduced among $\mathrm{mCRC}$ patients who experienced PTR or had intact primary tumour (IPT). All of them had the primary tumour tissue genotyping tested for RAS (KRAS and NRAS) gene mutations. The tumour-related overall survival (OS) time and progression-free survival (PFS) time was estimated. From January 2011 to June $2014,421 \mathrm{mCRC}$ patients with asymptomatic, unresectable, metastatic disease were enrolled in this study. Among them, 282 patients underwent PTR and 139 patients had IPT. Results: The mutation rate of RAS was $53.8 \%$ (221/411). With a median followed-up time of 46.5 months, the overall survival time of $m C R C$ patients harboring wtRAS or mtRAS was 28.0 versus 22.0 months $(p=0.043)$ in PTR group and was 21.6 versus 17.8 months $(p=0.071)$ in IPT groups. A Multivariate regression analysis suggested that RAS gene $(p=0.039, \mathrm{HR}=1.288,95 \% \mathrm{CI}$ [1.072 2.911]), metastatic organ number $(p=0.033, H R=3.091,95 \% C I$ [1.090 5.755]) and systemic therapy response $(p=0.019, H R=0.622,95 \% C I$ [0.525 0.811]) were independent prognostic factors in PTR population. Conclusion: We found that wild-type RAS gene was a favorable factor for the asymptomatic unresectable $\mathrm{mCRC}$ patients experiencing PTR.
\end{abstract}

L. Liang and J. Tian contributed equally to this work.

(C) 2018 The Author(s)

Published by S. Karger AG, Basel 


\section{Cellular Physiology Cell Physiol Biochem 2018;50:768-782 \begin{tabular}{l|l} 
DOI: 10.1159/000494242 & $\begin{array}{l}\text { O } 2018 \text { The Author(s). Published by S. Karger AG, Basel } \\
\text { www.karger.com/cpb }\end{array}$ \\
\hline aublished onnine: 12 October 2018
\end{tabular}}

Liang et al.: RAS Mutations in the Prognosis of Mcrc Patients

\section{Introduction}

Colorectal cancer (CRC) is a common type of malignant tumour worldwide. Almost $20 \%$ $-25 \%$ of patients with CRC have metastatic disease at the time of initial diagnosis and $75 \%$ $90 \%$ of them were unresectable [1]. The median survival time of metastatic colorectal cancer (mCRC) patients presenting with unresectable distant metastasis was about 5 months with best supportive care [2,3].

In general, the purpose of primary tumour resection (PTR) is to prevent or treat colon or rectal primary tumour related complications, such as intestinal obstruction, acute significant bleeding, perforation and systemic chemotherapy related complications brought from new molecular target therapy drugs (e.g. anti-angiogenesis monoclonal antibody) for colorectal cancer patients who had unresectable metastatic lesions [4]. But, considerable retrospective studies have suggested that CRC patients with asymptomatic primary tumour as well as synchronous unresectable metastases who underwent PTR had significantly longer survival time compared to those who only received palliative systemic chemotherapy [5-7]. A series of perspective clinical trials have been carried out to verify the survival superiority of PTR in this setting, such as CAIRO4 [NCT01606098], SYNCHRONOUS [ISRCTN30964555] and Korean trial [NCT01978249] [8-10].

It is crucial that oncologists should make a decision for asymptomatic unresectable mCRC patients who are most likely to be benefited from the non-curative resection of primary tumour. Dorajoo SR et al. [11] reported that clinical features, such as advanced age, poorly differentiated tumour, metastasis to liver, lung and bone, carcinomatosis, hypoalbuminaemia and elevated carcinoembryonic antigen levels, could significantly shorten post-operative survival of PTR. DeMestier et al. [12] summarized that other clinicpathological characteristics, including WHO-PS score, primary tumour site, chemotherapy regimen, liver metastasis burden and extra-hepatic metastatic disease were also the independent prognostic factors. Turner $\mathrm{N}$ et al. [13] suggested that systemic inflammation was also a negative factor form CRC patient who experience PTR.

The genetic features classification is regarded as basis for personalized therapy in mCRC in recent years, yet only RAS (KRAS, NRAS) and BRAF mutation have so far been wildly accepted as the biomarkers tools in clinical practice to help doctors administrate right therapy for right patients at the right time. The utility of BRAF gene as a biomarker is limited because BRAF V600E mutation rate is about $5 \%$ to $9 \%$ among colorectal cancer [14]. The RAS mutations prevalence (exon 2 and non-exon 2 of KRAS and NRAS) ranges from $50 \%$ to $60 \%$ [15] and have been taken as a predictive marker of resistance to EGFR blockage target therapy in conventional practice use [16]. RAS mutations may also have negative impact on patients' prognosis in metastatic setting. Osumi $\mathrm{H}$ et al. [17] evaluate the relationship between RAS mutations and clinical survival outcomes after mastectomy in mCRC patients and demonstrated that mutant-type RAS (mtRAS) was associated with shorter overall survival time. Waring P et al. [18] suggested that KRAS mutations in mCRC predispose to aggressive biology and possibly selection for therapy resistant clones. Payandeh M et al. [19] reported that there was a significant difference of KRAS codons mutations for survival.

At the present time, there are no biomarkers so far that have been identified to be a favorable predictor of PTR clinical survival outcome. Whether RAS mutation is related to a worse survival even if $\mathrm{MCRC}$ patients presented asymptomatic disease and undergo PTR remains uncertain. Therefore, we designed this retrospective observational study, trying to examine the impact on clinical survival among the population of mCRC patients who experienced PTR and verify the prognostic value to determine the subpopulation who would be really benefited from the non-curative surgery procedure. 


\title{
Cellular Physiology Cell Physiol Biochem 2018;50:768-782 \begin{tabular}{l|l} 
DOI: 10.1159/000494242 & $\begin{array}{l}\text { O 2018 The Author(s). Published by S. Karger AG, Basel } \\
\text { www.karger.com/cpb }\end{array}$
\end{tabular}
}

Liang et al.: RAS Mutations in the Prognosis of Mcrc Patients

\author{
Materials and Methods
}

Patients

A non-randomized retrospective cohort observation study was deduced among a series of consecutive metastatic colorectal patients. Some of these mCRC patients (1) presented asymptomatic primary and unresectable metastatic diseases needed no immediate surgery intervention or other treatments, (2) required positive treatment and (3) received the treatment plan made by physicians and surgeons together, then they underwent primary tumour resection. If the patients were unwilling to experience the surgical resection, they would accept palliative systemic chemotherapy with intact primary tumour (IPT) all the time. All of the patients had the primary tumour tissue genotyping tested for RAS (KRAS and NRAS gene) mutations. The protocol was approved by the ethics committee (ethics committee of Zhongshan Hospital of Fudan University, Shanghai), and written informed consent was obtained from all study participants. All methods were performed in accordance with the relevant guidelines.

\section{RAS mutations analysis}

Tumour specimen was from formalin-fixed, paraffin-embedded resected primary tumour mass or biopsy tissue under endoscopy. DNA extracted by FFPE QIAGNE kit and RAS statues were analyzed by the amplification refractory mutation system (ARMS). KRAS mutations referred to exon 2 (codons 12, 13), exon 3 (codons 59, 176,181), exon 4 (codons 146,117). NRAS mutations referred to exon 2 (codons 12, 13), exon 3 (codons 59, 61), exon 4 (codons 146, 117).

\section{Assessment measure of treatment outcomes}

The patients were radiologically examined every six to eight weeks to assess treatment response according to clinical guidelines, such as the NCCN guideline, which recommend abdominopelvic computed tomography (CT) scans, liver magnetic resonance imagines (MRI), and chest X-rays. If chest X-rays indicated metastatic disease in the lungs, chest CT scans were performed to confirm the diagnosis. The assessments were performed immediately if clinical signs indicated disease progression. A complete CBC with differentials and chemistry profiles was performed prior to each cycle. Objective tumour responses were measured according to the RECIST 1.1 criterion, including complete response (CR), partial response (PR), stable disease (SD) and progressive disease (PD). During treatment, hematological toxicities and nonhematological toxicities were assessed according to the National Cancer Institute Common Toxicity Criteria (NCI-CTC) scale version 3.0 [20]. Progression free survival (PFS) time was calculated from the initial of the treatment until disease first progression and overall survival (OS) time was referred to time until the date of death or last follow-up.

\section{Statistical analysis}

The statistical analyses were conducted with software of SPSS (version 12.0). Clinical characteristics were examined using the Chi squared test or Fisher's exact probability test. Time-related parameters were evaluated using the Kaplan-Meier method and were compared by Log-rank test.

\section{Results}

The prevalence of RAS gene mutation

From January 2011 to June 2014, a total of 1029 metastatic colorectal patients were first diagnosed and treated at the Department of Medical Oncology of Zhongshan Hospital affiliated to Fudan University. Finally, there were $421 \mathrm{mCRC}$ patients were enrolled in this study, with 282 cases underwent primary tumour resection and 139 cases had intact primary tumour. Four hundred and eleven cases successfully genotyped for RAS gene mutations. There were 176 cases and 45 cases detected mutated-type KRAS (42.8\%) and NRAS (10.9\%). In addition, there were 24 cases with wild-type RAS detected mutated-type BRAF of V600E (24/411, 5.8\%). The date of PTR and IPT group was showed in Fig. 1, respectively. 




Fig. 1. The research flow chart of this study.

\section{Clinic pathological characteristics}

The clinic pathological characteristics, such as gender, age, performance status, primary tumour site, number of metastatic organs did not significantly differ between all the patients (Table 1), PTR and IPT groups (Table 2), wtRAS and mtRAS groups (Table 3).

\section{Tumour-related Survival analysis}

With a median time of 46.5 (12-51) months, three hundred and ninety (390/411 , 94\%) patients died of tumour. The progression free survival time and overall survival time of mCRC patients who underwent PTR or had IPT was 9.2 versus 7.7 months ( $p=$ 0.005 ) and 26.0 versus 15.5 months $(p=0.001$ ) (Fig. 2 ). The progression free survival time of mCRC patients who underwent PTR or had IPT with wtRAS or mtRAS were 10.8 versus 8.5 months $(p=0.059), 7.9$ versus 6.8 months $(p=0.893)$ (Fig. 3). The overall-survival time of mCRC patients who underwent PTR or had IPT with wtRAS or mtRAS were 28.0 versus 21.6 months $(\mathrm{p}=0.024), 22.0$ versus 17.8 months $(\mathrm{p}=0.102)$ (Fig. 4). Then, the progression free survival time of mCRC patients who harbored wtRAS or mtRAS experienced PTR or IPT were 10.8 versus 7.9 months ( $p=0.032$ ), 8.5 versus 6.8 months ( $p=0.095$ ) (Fig. 5). The overall-survival time of mCRC patients who harbored wtRAS or mtRAS experienced PTR or IPT were 28.0 versus 22.0 months ( $p=0.043), 21.6$ versus 17.8 months ( $p=0.071)$ (Fig. 6), respectively. A statistical significance was proved by log-rank test. 
A Multivariate regression analysis

The clinical and pathological factors that affect the tumor-related progress free survival time and overall survival time among asymptomatic metastatic colorectal cancer patients in this study were compared by a method of multivariate regression (Table 4). Further analysis found that RAS gene ( $\mathrm{p}=0.039, \mathrm{HR}=1.288,95 \% \mathrm{CI}$ [1.072 2.911]), metastatic organ number $(\mathrm{p}=0.033, \mathrm{HR}=$ 3.091, 95\%CI [1.090 5.755]) and systemic therapy response $(\mathrm{p}=0.019, \mathrm{HR}=0.622$, 95\%CI [0.525 0.811]) were independent prognostic factors in PTR population (Table 5). Metastatic organ number ( $\mathrm{p}$ $=0.041, \mathrm{HR}=2.870,95 \% \mathrm{CI}$ [0.383 4.143]) and systemic therapy response $(\mathrm{p}=0.888$, HR $=0.043,95 \%$ CI [0.059 0.931]) were independent prognostic factors in IPT population (Table 6).

\section{Discussion}

The result of this study suggested that asymptomatic unresectable mCRC patients with wtRAS gene who experienced primary tumour resection had a longer overall survival time and progression free survival time than those had mtRAS gene. Inversely, mCRC patients with mtRAS gene had a similar survival outcome, regardless of whether they underwent primary tumour resection or had intact tumour all the time.

Regardless of a retrospective observational cohort study with small sample,
Table 1. The clinic pathological characteristics and treatment data of mCRC pts with asymptomatic unresectable diseases in this research * Left-Side Colon included rectum. ${ }^{\wedge}$ TACE referred to trans catheter chemoembolization. TAI referred to trans catheter arterial infusion

\begin{tabular}{|c|c|c|c|}
\hline Parameter & PTR group & IPT group & $\mathrm{p}$-value \\
\hline Patients Number & 278 & 133 & / \\
\hline Gender & & & 0.533 \\
\hline Male & $145(52 \%)$ & $65(49 \%)$ & \\
\hline Female & $133(48 \%)$ & $68(51 \%)$ & \\
\hline Mean Age & $57.2 \pm 11.9$ & $56.0 \pm 10.1$ & 0.713 \\
\hline Primary Tumor Site & & & 0.704 \\
\hline Right-Side Colon & $48(17 \%)$ & $25(6 \%)$ & \\
\hline Left-Side Colon* & $230(83 \%)$ & $108(94 \%)$ & \\
\hline Primary Tumour Size & & & 0.001 \\
\hline$<5 \mathrm{~cm}$ & $121(44 \%)$ & $82(62 \%)$ & \\
\hline$\geq 5 \mathrm{~cm}$ & $157(56 \%)$ & $51(38 \%)$ & \\
\hline Metastasis Organ & & & 0.746 \\
\hline Liver & $223(80 \%)$ & $102(77 \%)$ & \\
\hline Lung & $195(70 \%)$ & $104(78 \%)$ & \\
\hline Peritoneal site & $153(55 \%)$ & $77(58 \%)$ & \\
\hline Bone, etc & $83(30 \%)$ & $36(27 \%)$ & \\
\hline Number of Metastasis Organs & & & 0.706 \\
\hline Single & $185(67 \%)$ & $86(65 \%)$ & \\
\hline Multi & $93(33 \%)$ & $47(35 \%)$ & \\
\hline Metastatic Lesion Size & & & 0.780 \\
\hline$<5 \mathrm{~cm}$ & $181(65 \%)$ & $86(65 \%)$ & \\
\hline$\geq 5 \mathrm{~cm}$ & $93(33 \%)$ & $47(35 \%)$ & \\
\hline CEA Level & & & 0.787 \\
\hline$<5 \mu \mathrm{g} / \mathrm{ml}$ & $80(29 \%)$ & $40(30 \%)$ & \\
\hline$\geq 5 \mu \mathrm{g} / \mathrm{ml}$ & $198(71 \%)$ & $93(70 \%)$ & \\
\hline RAS Gene Type & & & 0.243 \\
\hline Wild type & $123(44 \%)$ & $67(50 \%)$ & \\
\hline Mutated type & $155(56 \%)$ & $66(50 \%)$ & \\
\hline First Line Chemo Regimen & & & 0.436 \\
\hline FOLFOX or CAPEOX & $137(49 \%)$ & $71(53 \%)$ & \\
\hline FOLFIRI & $141(51 \%)$ & $62(47 \%)$ & \\
\hline Second Line Chemo Regimen & & & 0.401 \\
\hline FOLFOX or CAPEOX & $123(44 \%)$ & $62(47 \%)$ & \\
\hline FOLFIRI & $125(45 \%)$ & $55(41 \%)$ & \\
\hline Capecitabine & $20(7 \%)$ & $14(11 \%)$ & \\
\hline None & $10(4 \%)$ & $2(1 \%)$ & \\
\hline Molecular Target Therapy & & & 0.001 \\
\hline Cetuximab & $77(28 \%)$ & $27(20 \%)$ & \\
\hline Bevacizumab & $72(26 \%)$ & $66(50 \%)$ & \\
\hline None & $129(46 \%)$ & $40(30 \%)$ & \\
\hline $\begin{array}{l}\text { Response to First Line } \\
\text { Therapy }\end{array}$ & & & 0.580 \\
\hline Partial Response & $50(18 \%)$ & $21(16 \%)$ & \\
\hline Stable Disease & $173(62 \%)$ & $80(60 \%)$ & \\
\hline Progressive Disease & $55(20 \%)$ & $32(24 \%)$ & \\
\hline Second Line Treatments & & & 0.554 \\
\hline Ablation & $140(50 \%)$ & $64(48 \%)$ & \\
\hline $\mathrm{TACE}$ or $\mathrm{TAI}^{\wedge}$ & $181(65 \%)$ & $93(70 \%)$ & \\
\hline Toxicity of Drugs Therapy & & & 0.204 \\
\hline Grade 1 to 2 & $228(82 \%)$ & $102(77 \%)$ & \\
\hline Over Grade 2 & $50(18 \%)$ & $31(23 \%)$ & \\
\hline Surgery Complications & & & / \\
\hline Infection & $15(5 \%)$ & / & \\
\hline Leakage & $15(5 \%)$ & / & \\
\hline \multicolumn{2}{|l|}{ Symptoms of Primary Tumour } & & / \\
\hline Haemorrhage & / & $9(7 \%)$ & \\
\hline Perforation & / & $3(2 \%)$ & \\
\hline Obstruction & / & $12(9 \%)$ & \\
\hline \multicolumn{3}{|c|}{ Salvage Treatment of Primary Tumour } & / \\
\hline Emergency Surgery & / & $7(5 \%)$ & \\
\hline Pelvic Radiotherapy & / & $12(9 \%)$ & \\
\hline Stent Plantation & / & $5(4 \%)$ & \\
\hline
\end{tabular}


Table 2. The clinic pathological characteristics and treatment data of mCRC pts with asymptomatic unresectable diseases who underwent primary tumour resection (PTR) or had intact primary tumour (IPT)

\begin{tabular}{|c|c|c|c|c|c|c|}
\hline \multirow{2}{*}{ Characteristics } & \multicolumn{3}{|c|}{ PTR group } & \multicolumn{3}{|c|}{ IPT group } \\
\hline & wtRAS & mtRAS & $\mathrm{p}$-value & wtRAS & mtRAS & $\mathrm{p}$-value \\
\hline Patients Number & 155 & 123 & l & 67 & 66 & / \\
\hline Gender & & & 0.213 & & & 0.663 \\
\hline Male & $86(56 \%)$ & $59(48 \%)$ & & $34(51 \%)$ & $31(47 \%)$ & \\
\hline Female & $69(44 \%)$ & $64(52 \%)$ & & $33(49 \%)$ & $35(53 \%)$ & \\
\hline Mean Age & $56.5 \pm 11.8$ & $58.1 \pm 12.1$ & 0.964 & $55.7 \pm 11.2$ & $56.3 \pm 9.3$ & 0.374 \\
\hline Primary Tumor Site & & & 0.808 & & & 0.532 \\
\hline Right-Side Colon & 26 (17\%) & $22(18 \%)$ & & $14(21 \%)$ & $11(17 \%)$ & \\
\hline Left-Side Colon & $129(83 \%)$ & $101(82 \%)$ & & $53(79 \%)$ & $55(83 \%)$ & \\
\hline Primary Tumour Size & & & 0.102 & & & 0.337 \\
\hline$<5 \mathrm{~cm}$ & $109(70 \%)$ & $75(61 \%)$ & & $44(66 \%)$ & $38(58 \%)$ & \\
\hline$\geq 5 \mathrm{~cm}$ & $46(30 \%)$ & $48(39 \%)$ & & $23(34 \%)$ & $28(42 \%)$ & \\
\hline Metastasis Organ & & & 0.019 & & & 0.645 \\
\hline Liver & $122(79 \%)$ & 101(82\%) & & $46(68 \%)$ & $56(85 \%)$ & \\
\hline Lung & $101(65 \%)$ & $94(76 \%)$ & & $50(74 \%)$ & $54(81 \%)$ & \\
\hline Peritoneal site & $69(45 \%)$ & $84(68 \%)$ & & $33(49 \%)$ & $44(67 \%)$ & \\
\hline Bone, etc & $30(19 \%)$ & $53(43 \%)$ & & $13(19 \%)$ & $23(35 \%)$ & \\
\hline Number of Metastasis Organs & & & 0.214 & & & 0.182 \\
\hline Single & $108(70 \%)$ & 77 (63\%) & & 47 (70\%) & $39(59 \%)$ & \\
\hline Multi & $47(30 \%)$ & $46(37 \%)$ & & $20(30 \%)$ & $27(41 \%)$ & \\
\hline Metastatic Lesion Size & & & 0.301 & & & 0.339 \\
\hline$<5 \mathrm{~cm}$ & $105(68 \%)$ & $76(62 \%)$ & & $43(64 \%)$ & $37(56 \%)$ & \\
\hline$\geq 5 \mathrm{~cm}$ & $50(32 \%)$ & $47(38 \%)$ & & $24(36 \%)$ & $29(44 \%)$ & \\
\hline CEA Level & & & 0.709 & & & 0.484 \\
\hline$<5 \mu \mathrm{g} / \mathrm{ml}$ & $46(30 \%)$ & $34(28 \%)$ & & $22(31 \%)$ & $18(27 \%)$ & \\
\hline$\geq 5 \mu \mathrm{g} / \mathrm{ml}$ & $109(70 \%)$ & $89(72 \%)$ & & $45(69 \%)$ & $48(73 \%)$ & \\
\hline First Line Treatment Regimer & & & 0.382 & & & 0.539 \\
\hline FOLFOX or CAPEOX $80(52 \%$ & & $57(46 \%)$ & & $34(51 \%)$ & $37(56 \%)$ & \\
\hline FOLFIRI & 75 (48\%) & $66(54 \%)$ & & $33(49 \%)$ & $29(44 \%)$ & \\
\hline Second Line Chemo Regimen & & & 0.569 & & & 0.554 \\
\hline FOLFOX or CAPEOX $63(41 \%$ & & $60(49 \%)$ & & $30(45 \%)$ & 32 (49\%) & \\
\hline FOLFIRI & 75 (48\%) & $50(41 \%)$ & & $31(46 \%)$ & $24(36 \%)$ & \\
\hline Capecitabine & $11(7 \%)$ & $9(7 \%)$ & & $5(8 \%)$ & $9(14 \%)$ & \\
\hline None & $6(4 \%)$ & $4(3 \%)$ & & $1(1 \%)$ & $1(1 \%)$ & \\
\hline Molecular Target Therapy & & & 0.001 & & & 0.001 \\
\hline Cetuximab & 77 (50\%) & $0(0 \%)$ & & 27 (40\%) & $0(0 \%)$ & \\
\hline Bevacizumab & $33(21 \%)$ & $39(32 \%)$ & & $22(33 \%)$ & $44(67 \%)$ & \\
\hline None & $45(29 \%)$ & $84(68 \%)$ & & $18(27 \%)$ & $22(33 \%)$ & \\
\hline Response to First Line Thera & & & & 0.113 & & 0.418 \\
\hline Partial Response & $31(20 \%)$ & $19(15 \%)$ & & $12(18 \%)$ & $9(14 \%)$ & \\
\hline Stable Disease & $100(65 \%)$ & $73(59 \%)$ & & $42(64 \%)$ & $38(57 \%)$ & \\
\hline Progressive Disease & $24(15 \%)$ & $31(26 \%)$ & & $13(18 \%)$ & $19(29 \%)$ & \\
\hline Second Line Treatments & & & 0.183 & & & 0.303 \\
\hline Ablation & 77 (50\%) & $63(51 \%)$ & & $27(40 \%)$ & $37(56 \%)$ & \\
\hline TACE or TAI^ & $86(55 \%)$ & $95(77 \%)$ & & $47(70 \%)$ & $46(70 \%)$ & \\
\hline Toxicity of Drugs Therapy & & & 0.555 & & & 0.570 \\
\hline Grade 1 to 2 & $129(83 \%)$ & $99(80 \%)$ & & $50(75 \%)$ & $52(79 \%)$ & \\
\hline Over Grade 2 & $26(17 \%)$ & $24(20 \%)$ & & $17(25 \%)$ & $14(11 \%)$ & \\
\hline Surgery Complications & & & 0.713 & & & / \\
\hline Infection & $9(6 \%)$ & $6(5 \%)$ & & / & / & \\
\hline Leakage & $8(5 \%)$ & $7(6 \%)$ & & / & / & \\
\hline Symptoms of Primary Tumou & & & & & & / \\
\hline Haemorrhage & / & / & & $3(4 \%)$ & $6(9 \%)$ & \\
\hline Perforation & / & / & & $1(1 \%)$ & $2(3 \%)$ & \\
\hline Obstruction & / & / & & $7(10 \%)$ & $5(8 \%)$ & \\
\hline Salvage Treatment of Primary & y Tumour & & & & & / \\
\hline Emergency & / & I & & $4(6 \%)$ & $3(2 \%)$ & \\
\hline Pelvic Radiotherapy & / & / & & $6(9 \%)$ & $6(4 \%)$ & \\
\hline Stent Plantation & 1 & 1 & & $3(5 \%)$ & $2(1 \%)$ & \\
\hline
\end{tabular}


Table 3. The clinicopathological characteristics and treatment data of mCRC pts with asymptomatic unresectable diseases who underwent primary tumour resection (PTR) or had intact primary tumour (IPT)

\begin{tabular}{|c|c|c|c|c|c|c|}
\hline \multirow{2}{*}{ Parameter } & \multicolumn{3}{|c|}{ wtRAS Group } & \multicolumn{2}{|c|}{ mtRAS Group } & \multirow[b]{2}{*}{$\mathrm{p}$-value } \\
\hline & PTR & IPT & $p$-value & PTR & IPT & \\
\hline Patients Number & 155 & 67 & 1 & 123 & 66 & \multirow{4}{*}{0.896} \\
\hline \multicolumn{3}{|l|}{ Gender } & 0.516 & & & \\
\hline Male & $86(56 \%)$ & $34(51 \%)$ & & $59(48 \%)$ & $31(47 \%)$ & \\
\hline Female & $69(44 \%)$ & $33(49 \%)$ & & $64(52 \%)$ & $35(53 \%)$ & \\
\hline Mean Age & $56.5 \pm 11.8$ & $55.7 \pm 11.2$ & 0.875 & $58.1 \pm 12.1$ & $56.3 \pm 9.3$ & \multirow{4}{*}{$\begin{array}{l}0.521 \\
0.833\end{array}$} \\
\hline \multicolumn{2}{|c|}{ Primary Tumor Site } & & 0.463 & & & \\
\hline Right-Side Colon & $26(17 \%)$ & 14 (21\%) & & $22(18 \%)$ & $11(17 \%)$ & \\
\hline Left-Side Colon & $129(83 \%)$ & $53(79 \%)$ & & $101(82 \%)$ & $55(83 \%)$ & \\
\hline \multicolumn{2}{|c|}{ Primary Tumour Size } & & 0.492 & & 0.650 & \multirow{8}{*}{0.816} \\
\hline$<5 \mathrm{~cm}$ & $109(70 \%)$ & $44(66 \%)$ & & 75 (61\%) & 38 (58\%) & \\
\hline$\geq 5 \mathrm{~cm}$ & $46(30 \%)$ & $23(34 \%)$ & & $48(39 \%)$ & $28(42 \%)$ & \\
\hline \multicolumn{2}{|l|}{ Metastasis Organ } & & 0.701 & & & \\
\hline Liver & $122(79 \%)$ & $46(68 \%)$ & & $101(82 \%)$ & $56(85 \%)$ & \\
\hline Lung & $101(65 \%)$ & $50(74 \%)$ & & $94(76 \%)$ & $54(81 \%)$ & \\
\hline Peritoneal site & $69(45 \%)$ & $33(49 \%)$ & & $84(68 \%)$ & $44(67 \%)$ & \\
\hline Bone, etc & $30(19 \%)$ & $13(19 \%)$ & & $53(43 \%)$ & $23(35 \%)$ & \\
\hline \multicolumn{2}{|c|}{ Number of Metastasis } & & 0.944 & & 0.496 & \\
\hline Single & $108(70 \%)$ & $47(70 \%)$ & & 77 (63\%) & $39(59 \%)$ & \\
\hline Multi & $47(30 \%)$ & $20(30 \%)$ & & $46(37 \%)$ & 27 (41\%) & \\
\hline \multicolumn{2}{|c|}{ Metastatic Lesion Size } & & 0.605 & & 0.444 & \\
\hline$<5 \mathrm{~cm}$ & $105(68 \%)$ & $43(64 \%)$ & & $76(62 \%)$ & $37(56 \%)$ & \\
\hline$\geq 5 \mathrm{~cm}$ & $50(32 \%)$ & $24(36 \%)$ & & $47(38 \%)$ & $29(44 \%)$ & \\
\hline \multicolumn{2}{|l|}{ CEA Level } & & 0.639 & & & \multirow[t]{3}{*}{0.957} \\
\hline$<5 \mu \mathrm{g} / \mathrm{ml}$ & $46(30 \%)$ & $22(31 \%)$ & & $34(28 \%)$ & $18(27 \%)$ & \\
\hline$\geq 5 \mu \mathrm{g} / \mathrm{ml}$ & $109(70 \%)$ & $45(69 \%)$ & & $89(72 \%)$ & $48(73 \%)$ & \\
\hline First Line Chemo & imen & & 0.906 & & & 0.203 \\
\hline FOLFOX or CAPE & $0(52 \%)$ & $34(51 \%)$ & & $57(46 \%)$ & $37(56 \%)$ & \\
\hline FOLFIRI & 75 (48\%) & $33(49 \%)$ & & $66(54 \%)$ & $29(44 \%)$ & \\
\hline Second Line Cher & egimen & & 0.781 & & & 0.477 \\
\hline FOLFOX or CAPE & $3(41 \%)$ & $30(45 \%)$ & & $60(49 \%)$ & $32(49 \%)$ & \\
\hline FOLFIRI & 75 (48\%) & 31 (46\%) & & $50(41 \%)$ & $24(36 \%)$ & \\
\hline Capecitabine & $11(7 \%)$ & $5(8 \%)$ & & $9(7 \%)$ & $9(14 \%)$ & \\
\hline None & $6(4 \%)$ & $1(1 \%)$ & & $4(3 \%)$ & $1(1 \%)$ & \\
\hline Molecular Target & apy & & 0.176 & & & 0.001 \\
\hline Cetuximab & 77 (50\%) & 27 (40\%) & & $0(0 \%)$ & 0 & \\
\hline Bevacizumab & $33(21 \%)$ & $22(33 \%)$ & & $39(32 \%)$ & $44(67 \%)$ & \\
\hline None & $45(29 \%)$ & $18(27 \%)$ & & $84(68 \%)$ & $22(33 \%)$ & \\
\hline Response to Firs & e Therapy & & 0.755 & & & 0.850 \\
\hline Partial Response & $31(20 \%)$ & 12 (18\%) & & $19(15 \%)$ & $9(14 \%)$ & \\
\hline Stable Disease & $100(65 \%)$ & $42(64 \%)$ & & 73 (59\%) & $38(57 \%)$ & \\
\hline $\begin{array}{l}\text { Progressive } \\
\text { Disease }\end{array}$ & $24(15 \%)$ & 13 (18\%) & & $31(26 \%)$ & $19(29 \%)$ & \\
\hline Second Line Trea & & & 0.122 & & & 0.481 \\
\hline Ablation & 77 (50\%) & 27 (40\%) & & $63(51 \%)$ & $37(56 \%)$ & \\
\hline TACE or TAI^ ${ }^{\wedge}$ & $86(55 \%)$ & 47 (70\%) & & $95(77 \%)$ & $46(70 \%)$ & \\
\hline Toxicity of Drugs & rapy & & 0.137 & & & 0.781 \\
\hline Grade 1 to 2 & $129(83 \%)$ & $50(75 \%)$ & & 99 (80\%) & $52(79 \%)$ & \\
\hline Over Grade 2 & $26(17 \%)$ & 17 (25\%) & & $24(20 \%)$ & $14(11 \%)$ & \\
\hline Surgery Complic & & & l & & & I \\
\hline Infection & $9(6 \%)$ & / & & $6(5 \%)$ & / & \\
\hline Leakage & $8(5 \%)$ & / & & $7(6 \%)$ & / & \\
\hline Symptoms of Pri & Tumour & & / & & & / \\
\hline Haemorrhage & / & $3(4 \%)$ & & / & $6(9 \%)$ & \\
\hline Perforation & 1 & $1(1 \%)$ & & / & $2(3 \%)$ & \\
\hline Obstruction & / & $7(10 \%)$ & & / & $5(8 \%)$ & \\
\hline Salvage Treatmer & Primary Tun & & & & & / \\
\hline Emergency & / & $4(6 \%)$ & & / & $3(2 \%)$ & \\
\hline $\begin{array}{l}\text { Pelvic } \\
\text { Radiotherany }\end{array}$ & / & $6(9 \%)$ & & / & $6(4 \%)$ & \\
\hline Stent Plantation & 1 & $3(5 \%)$ & & / & $2(1 \%)$ & \\
\hline
\end{tabular}




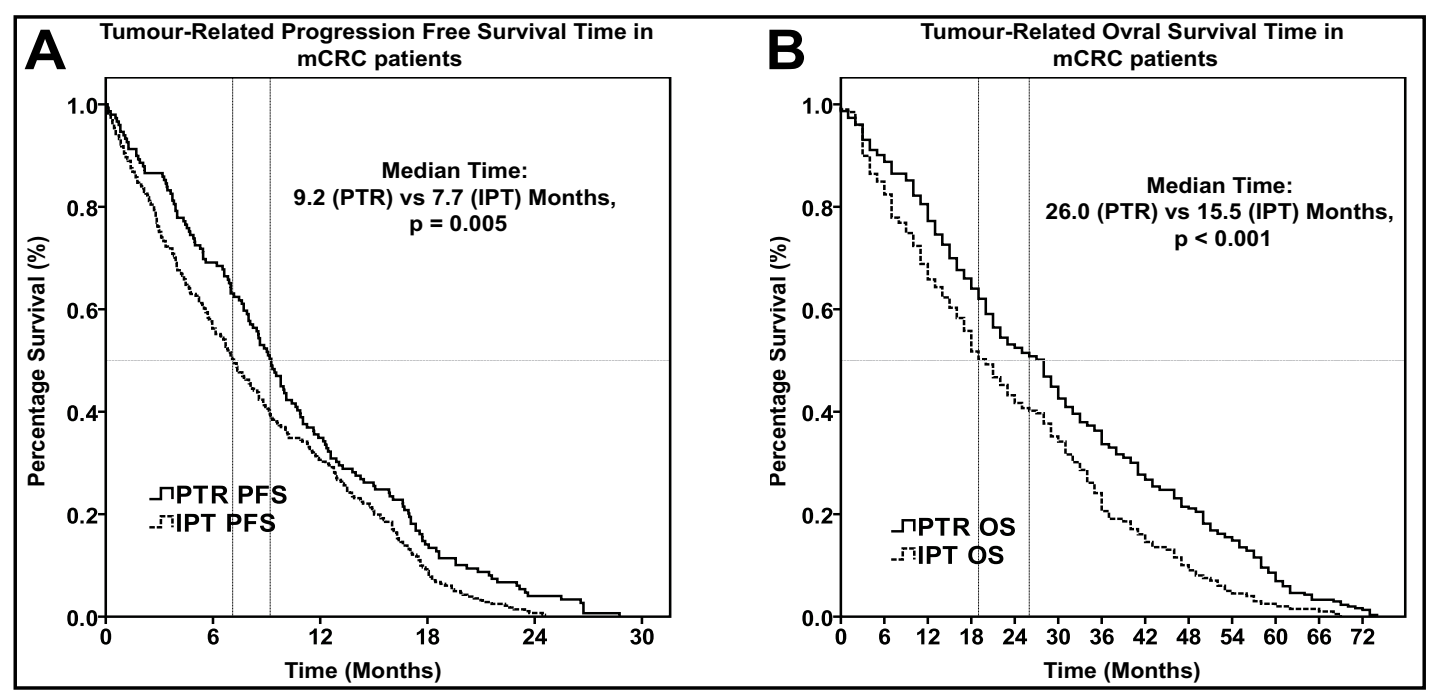

Fig. 2. Survival outcomes were analyzed for tumour-related progression free survival time (PFS) and overall survival time (OS) between the PTR group and IPT group by Kaplan - Meier method. With a median followed-up time of 46.5 months, the PFS and OS time of mCRC patients who underwent PTR or had IPT were 9.2 versus 7.7 months ( $p=0.005), 26.0$ versus $15.5(p=0.001)$ respectively. A statistical significance was proved by log-rank test.

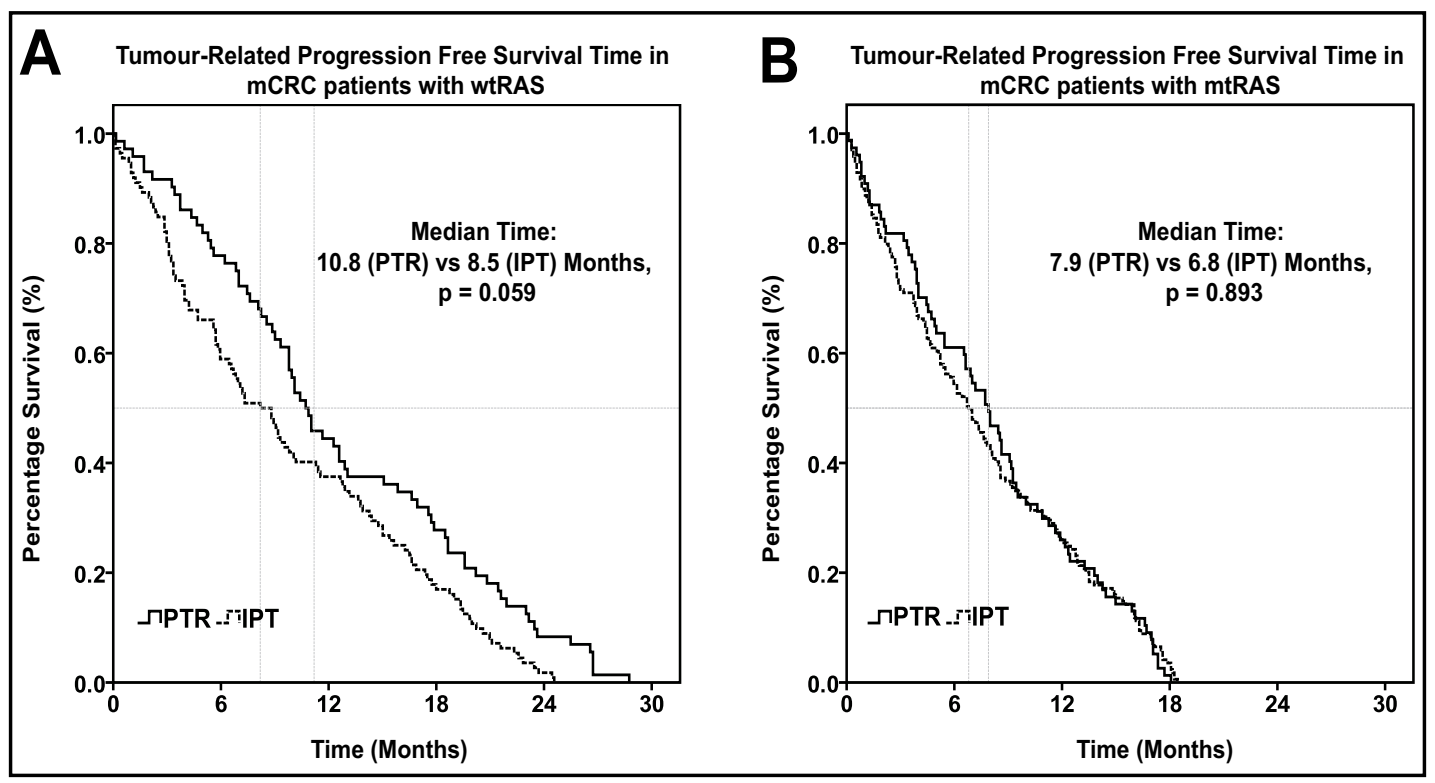

Fig. 3. Survival outcomes were analyzed for tumour-related progression free survival time (PFS) between the wtRAS group (A) and mtRAS group (B) by Kaplan - Meier method. The PFS time of mCRC patients who underwent PTR or had IPT with wtRAS or mtRAS were10.8 versus 8.5 months ( $p=0.059), 7.9$ versus 6.8 months ( $p=0.893)$, respectively. A statistical significance was proved by log-rank test. 


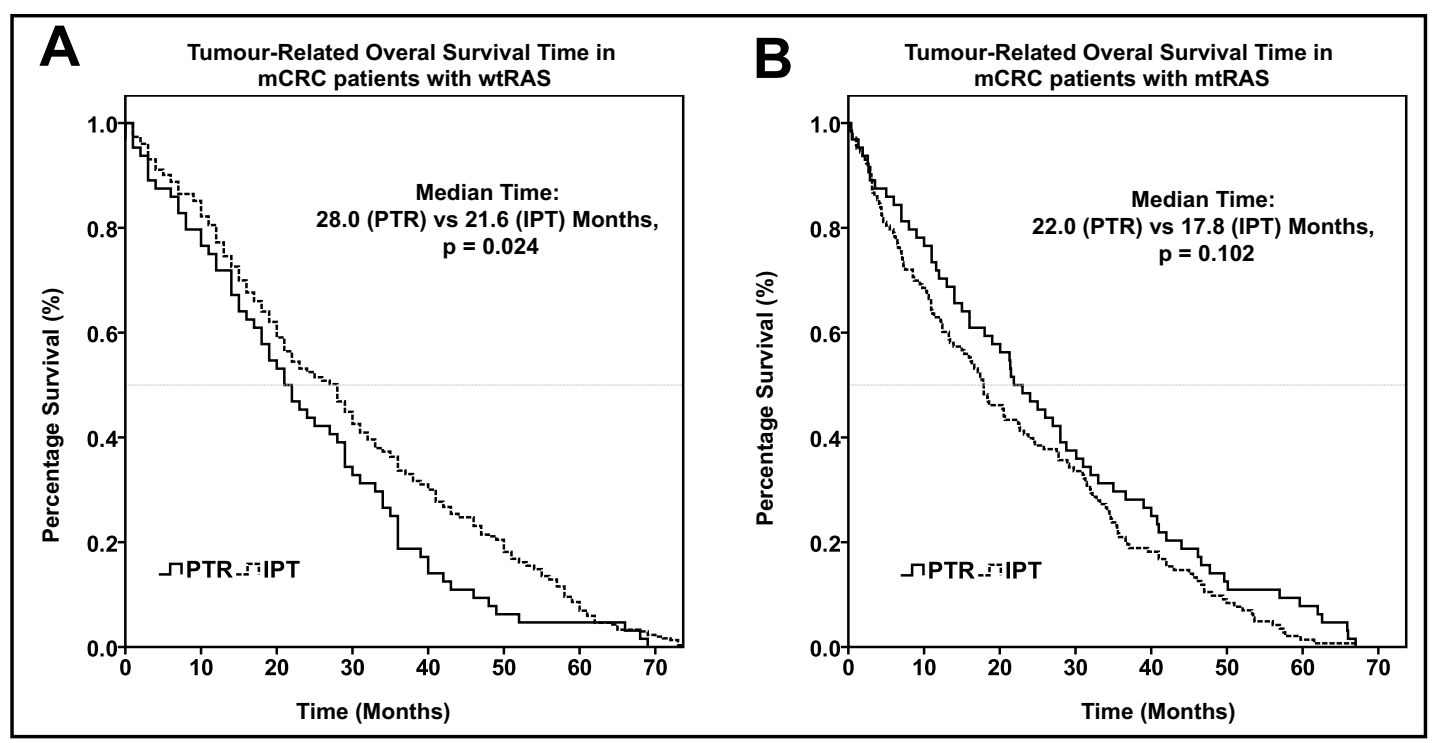

Fig. 4. Survival outcomes were analyzed for tumour-related overall survival time (OS) between the wtRAS group (A) and mtRAS group (B) by Kaplan - Meier method. The OS time of mCRC patients who underwent PTR or had IPT with wtRAS or mtRAS were 28.0 versus 21.6 months $(p=0.024), 22.0$ versus 17.8 months $(\mathrm{p}=0.102)$, respectively. A statistical significance was proved by log-rank test.
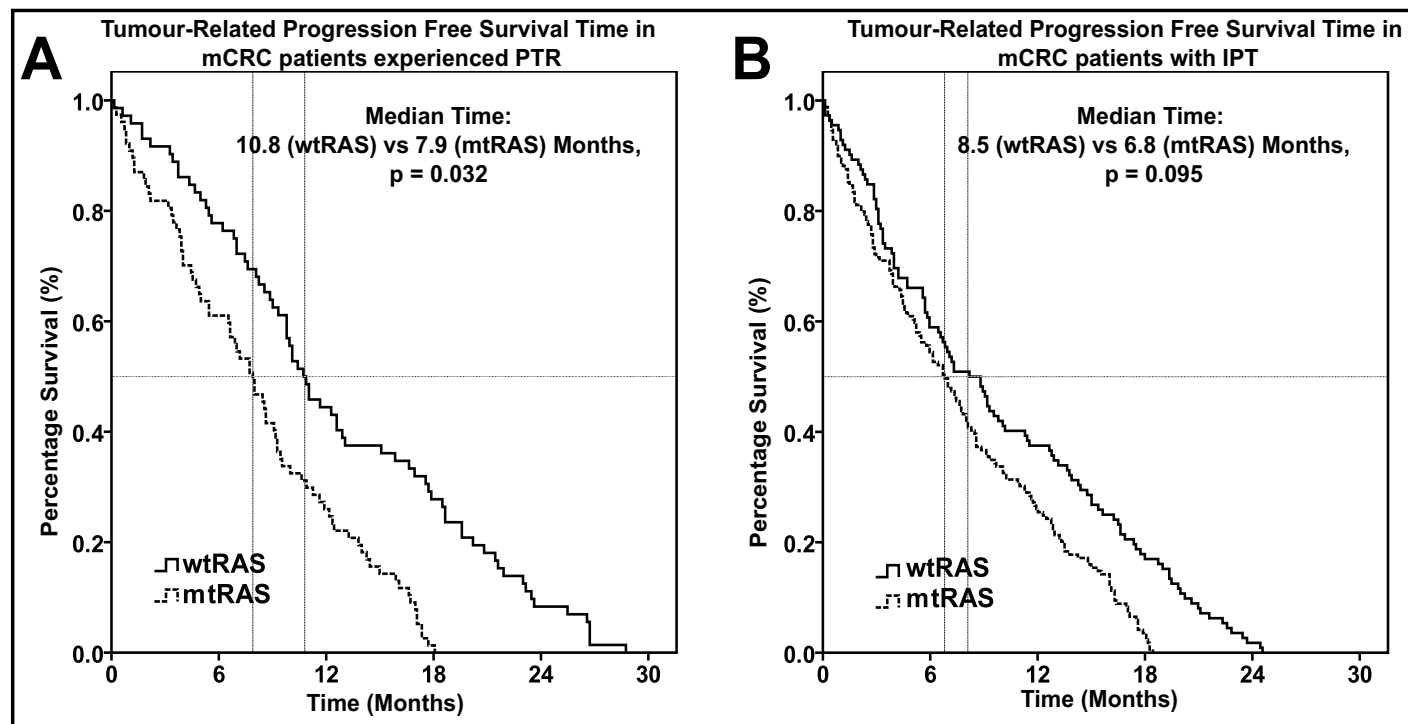

Fig. 5. Survival outcomes were analyzed for tumour-related progression free survival time (PFS) between the PTR group (A) and IPT (B) by Kaplan - Meier method. The PFS time of mCRC patients who harbored wtRAS or mtRAS experienced PTR or IPT were 10.8 versus 7.9 months ( $p=0.032), 8.5$ versus 6.8 months ( $p$ = 0.095), respectively. A statistical significance was proved by log-rank test. 


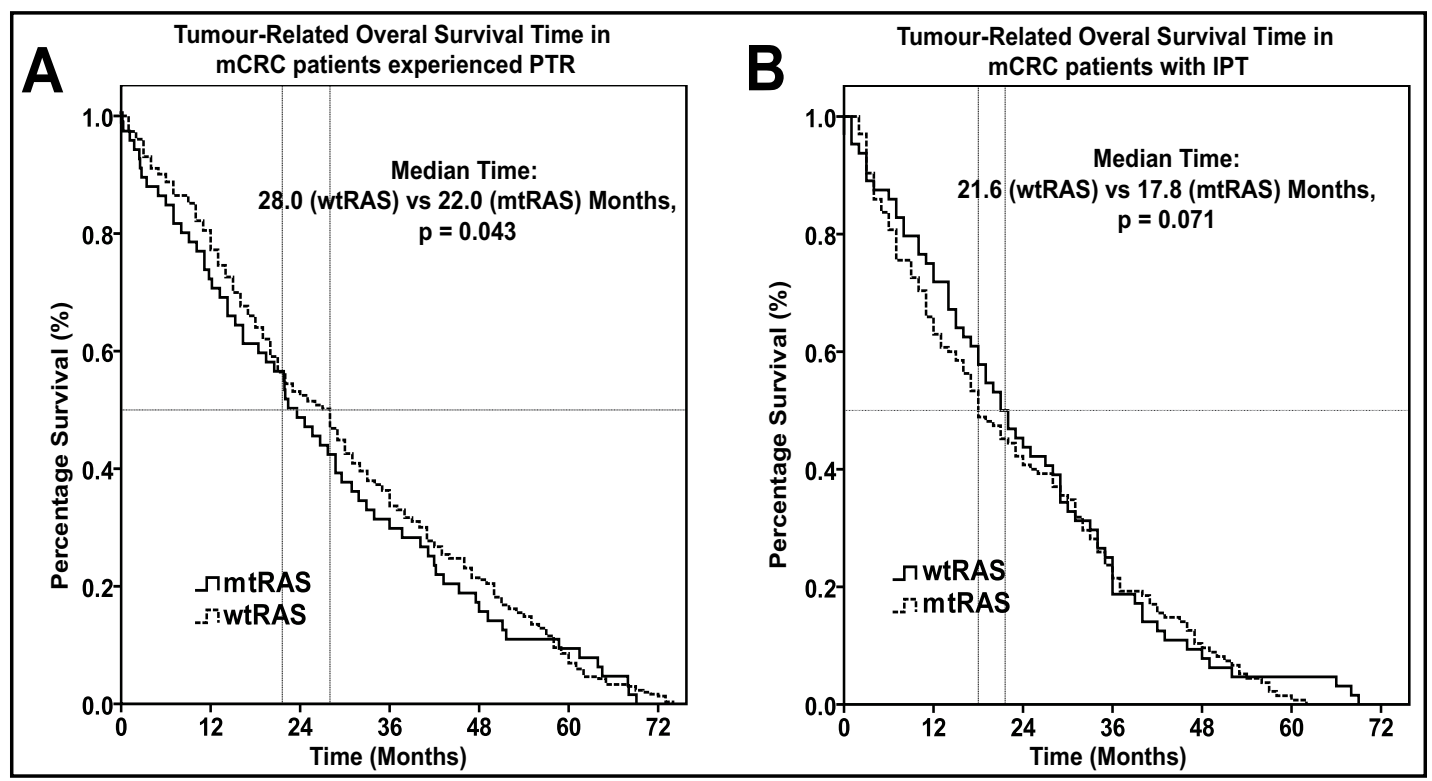

Fig. 6. Survival outcomes were analyzed for tumour-related overall survival time (OS) between the PTR group (A) and IPT (B) by Kaplan - Meier method. The OS time of mCRC patients who harbored wtRAS or mtRAS experienced PTR or IPT were 28.0 versus 22.0 months $(\mathrm{p}=0.043), 21.6$ versus 17.8 months $(\mathrm{p}=$ $0.071)$, respectively. A statistical significance was proved by log-rank test.

Table 4. A Multi-factor regression analysis was deduced on the clinical and pathological factors that affect the tumor-related progress free survival time and overall survival time among asymptomatic metastatic colorectal cancer patients in this study (All the cases)

\begin{tabular}{|c|c|c|c|c|c|c|}
\hline \multirow{3}{*}{$\begin{array}{l}\text { mCRC Patients with Asymptomatic Unresectable Diseases } \\
\text { Clinical-pathological Characteristics }\end{array}$} & \multicolumn{6}{|c|}{ Multi-Factors Cox Regression Analysis } \\
\hline & \multicolumn{3}{|c|}{ Tumour-Related Progresssion Free Survival } & \multicolumn{3}{|c|}{ Tumour-Related Overall Survival } \\
\hline & Hazards Rates & $95 \%$ Confidence Interval & $\mathrm{p}$-value & Hazards Rates & 95\% Confidence Interval & $\mathrm{p}$-value \\
\hline Gender (Male/Female) & 0.552 & $0.984-1.730$ & 0.442 & 0.498 & $0.893-1.902$ & 0.587 \\
\hline Mean Age & 1.298 & $0.734-2.269$ & 0.321 & 1.300 & $0.634-2.667$ & 0.474 \\
\hline PS Score $(0 / 1-2)$ & 0.732 & $0.381-1.874$ & 0.373 & 0.633 & $0.282-2.074$ & 0.438 \\
\hline RAS Status (Wild/ Mutated) & 1.592 & $0.221-3.021$ & 0.411 & 1.219 & $0.093-1.987$ & 0.328 \\
\hline Primary Tumor Size $(<5 \mathrm{~cm} / \geq 5 \mathrm{~cm})$ & 1.231 & $1.007-2.189$ & 0.035 & 1.309 & $0.782-2.013$ & 0.413 \\
\hline Primary Tumor Site (right/left) & 0.732 & $0.480-1.538$ & 0.612 & 0.876 & $0.801-2.182$ & 0.711 \\
\hline Metastasis Organ & 1.897 & $0.258-2.981$ & 0.098 & 2.134 & $0.981-3.214$ & 0.104 \\
\hline Number of Metastasis Organs (single-/multi-) & 2.294 & $1.532-3.012$ & 0.029 & 2.189 & $1.318-3.134$ & 0.024 \\
\hline Metastatic Lesion Size $(<5 \mathrm{~cm} / \geq 5 \mathrm{~cm})$ & 3.271 & $0.624-4.174$ & 0.851 & 3.037 & $0.671-5.371$ & 0.531 \\
\hline PTR / IPT & 0.712 & $0.451-1.321$ & 0.081 & 0.821 & $0.698-0.909$ & 0.032 \\
\hline First Line Chemotherapy Regimen & 0.987 & $0.109-3.012$ & 0.752 & 0.827 & $0.201-2.125$ & 0.289 \\
\hline Anti-EGFR Target Therapy (Yes/No) & 0.873 & $0.501-2.917$ & 0.082 & 0.782 & $0.491-1.864$ & 0.067 \\
\hline Anti-VGFR Target Therapy (Yes/No) & 0.791 & $0.201-1.982$ & 0.109 & 0.821 & $0.581-2.182$ & 0.069 \\
\hline First-Line Therapy Response (CR/SD/PD) & 3.912 & $1.891-5.098$ & 0.038 & 2.039 & $1.082-2.983$ & 0.023 \\
\hline Any Adverse Events from Systemic Therapy & 1.253 & $0.672-2.129$ & 0.528 & 1.513 & $0.589-3.862$ & 0.677 \\
\hline Second Line Treatments & 0.954 & $0.561-1.842$ & 0.657 & 0.814 & $0.421-1.928$ & 0.824 \\
\hline Complications of Surgery & 1.021 & $0.251-2.021$ & 0.133 & 1.902 & $0.581-2.873$ & 0.431 \\
\hline
\end{tabular}

Table 5. A Multi-factor regression analysis was deduced on the clinical and pathological factors that affect the tumor-related progress free survival time and overall survival time among asymptomatic metastatic colorectal cancer patients who experienced primary tumor resection (PTR group)

\begin{tabular}{|c|c|c|c|c|c|c|}
\hline \multirow{3}{*}{$\begin{array}{l}\text { mCRC Patients with Asymptomatic Diseases } \\
\text { Experienced Primary Tumor Resection }\end{array}$} & \multicolumn{6}{|c|}{ Multi-Factors Cox Regression Analysis } \\
\hline & \multicolumn{3}{|c|}{ Tumour-Related Progresssion Free Survival } & \multicolumn{3}{|c|}{ Tumour-Related Overall Survival } \\
\hline & Hazards Rates & 95\% Confidence Interval & $\mathrm{p}$-value & Hazards Rates & 95\% Confidence Interval & $\mathrm{p}$-value \\
\hline Gender (Male/Female) & 0.673 & $0.724-1.530$ & 0.342 & 0.921 & $0.801-4.902$ & 0.744 \\
\hline Mean Age & 1.441 & $0.186-1.548$ & 0.164 & 1.927 & $0.476-3.808$ & 0.358 \\
\hline PS Score $(0 / 1-2)$ & 0.815 & $0.716-2.141$ & 0.731 & 0.790 & $0.529-3.074$ & 0.689 \\
\hline RAS Status (Wild/ Mutated) & 1.828 & $1.209-3.001$ & 0.045 & 1.288 & $1.072-2.911$ & 0.039 \\
\hline Primary Tumor Size $(<5 \mathrm{~cm} / \geq 5 \mathrm{~cm})$ & 2.313 & $1.102-4.902$ & 0.101 & 1.991 & $1.812-5.328$ & 0.277 \\
\hline Primary Tumor Site (right/left) & 0.932 & $0.167-2.580$ & 0.322 & 0.894 & $0.301-3.021$ & 0.591 \\
\hline Metastasis Organ & 2.981 & $0.812-3.616$ & 0.569 & 2.521 & $0.716-3.164$ & 0.422 \\
\hline Number of Metastasis (single-/multi-) & 2.523 & $1.022-4.072$ & 0.091 & 3.091 & $1.089-5.755$ & 0.033 \\
\hline Metastatic Lesion Size $(<5 \mathrm{~cm} / \geq 5 \mathrm{~cm})$ & 2.021 & $0.784-3.936$ & 0.085 & 1.988 & $0.751-3.331$ & 0.105 \\
\hline First Line Chemotherapy Regimen & 0.987 & $0.109-3.299$ & 0.896 & 0.899 & $0.488-2.789$ & 0.891 \\
\hline Anti-EGFR Target Therapy (Yes/No) & 0.744 & $0.288-1.917$ & 0.112 & 0.682 & $0.333-2.314$ & 0.081 \\
\hline Anti-VGFR Target Therapy (Yes/No) & 0.612 & $0.301-2.863$ & 0.210 & 0.533 & $0.281-1.903$ & 0.209 \\
\hline First-Line Therapy Response (CR/SD/PD) & 0.588 & $0.191-0.985$ & 0.025 & 0.622 & $0.525-0.811$ & 0.019 \\
\hline Any Adverse Events from Systemic Therapy & 2.513 & $0.992-4.219$ & 0.877 & 2.883 & $0.819-4.612$ & 0.711 \\
\hline Second Line Treatments & 0.852 & $0.368-2.164$ & 0.236 & 0.799 & $0.617-2.394$ & 0.236 \\
\hline Complications of Surgery & 3.214 & $0.593-5.891$ & 0.323 & 2.919 & $0.875-4.093$ & 0.531 \\
\hline
\end{tabular}


Table 6. A Multi-factor regression analysis was deduced on the clinical and pathological factors that affect the tumor-related progress free survival time and overall survival time among asymptomatic metastatic colorectal cancer patients who had intact primary tumor all the time (IPT group)

\begin{tabular}{|c|c|c|c|c|c|c|}
\hline \multirow{3}{*}{$\begin{array}{l}\text { mCRC Patients with Asymptomatic Diseases } \\
\text { and Intact Primary Tumor All the Time }\end{array}$} & \multicolumn{6}{|c|}{ Multi-Factors Cox Regression Analysis } \\
\hline & \multicolumn{3}{|c|}{ Tumour-Related Progresssion Free Survival } & \multicolumn{3}{|c|}{ Tumour-Related Overall Survival } \\
\hline & Hazards Rates & 95\% Confidence Interval & $\mathrm{p}$ value & Hazards Rates & 95\% Confidence Interval & $\mathrm{p}$ value \\
\hline Gender (Male/Female) & 0.494 & $0.684-2.302$ & 0.550 & 0.401 & $0.793-3.902$ & 0.609 \\
\hline Mean Age & 1.217 & $0.861-2.226$ & 0.229 & 1.007 & $0.536-2.417$ & 0.816 \\
\hline PS Score $(0 / 1-2)$ & 0.392 & $0.199-2.174$ & 0.637 & 0.794 & $0.182-2.342$ & 0.490 \\
\hline RAS Status (Wild/ Mutated) & 1.791 & $1.013-4.231$ & 0.211 & 1.691 & $1.023-3.287$ & 0.108 \\
\hline Primary Tumor Size $(<5 \mathrm{~cm} / \geq 5 \mathrm{~cm})$ & 2.312 & $1.807-3.189$ & 0.501 & 2.091 & $1.801-3.013$ & 0.233 \\
\hline Primary Tumor Site (right/left) & 0.772 & $0.180-1.989$ & 0.289 & 0.543 & $0.201-2.822$ & 0.288 \\
\hline Metastasis Organ & 2.310 & $0.814-4.326$ & 0.701 & 2.066 & $0.912-3.199$ & 0.622 \\
\hline Number of Metastasis (single-/multi-) & 3.143 & $1.328-6.842$ & 0.062 & 2.870 & $0.383-4.143$ & 0.041 \\
\hline Metastatic Lesion Size $(<5 \mathrm{~cm} / \geq 5 \mathrm{~cm})$ & 3.306 & $0.922-5.654$ & 0.566 & 2.944 & $0.841-4.299$ & 0.633 \\
\hline First Line Chemotherapy Regimen & 0.572 & $0.231-2.102$ & 0.578 & 0.732 & $0.144-3.217$ & 0.592 \\
\hline Anti-EGFR Target Therapy (Yes/No) & 0.633 & $0.083-0.890$ & 0.078 & 0.710 & $0.190-1.064$ & 0.059 \\
\hline Anti-VGFR Target Therapy (Yes/No) & 0.702 & $0.101-1.332$ & 0.093 & 0.821 & $0.181-2.182$ & 0.107 \\
\hline First-Line Therapy Response (CR/SD/PD) & 0.601 & $0.081-0.082$ & 0.039 & 0.888 & $0.059-0.931$ & 0.043 \\
\hline Second Line Treatments & 0.801 & $0.266-2.365$ & 0.091 & 0.784 & $0.311-3.317$ & 0.522 \\
\hline Any Adverse Events from Systemic Therapy & 2.899 & $0.988-6.223$ & 0.799 & 3.003 & $0.792-5.122$ & 0.880 \\
\hline
\end{tabular}

the results of this study suggested a significant difference and condition, which lacked reports.

There were no significant differences in baseline characteristics between the PTR and IPT group, such as clinic pathological features of primary and metastastic lesions, chemo- or molecular therapy regimens and response to systemic treatments. The similar RAS mutation prevalence and survival outcomes were found by this research group according to the literature reported in metastatic setting.

A recent pooled analysis suggested that RAS mutation (including KRAS and NRAS) prevalence in mCRC patients was about 55.9\%, with a distribution as: $42.6 \%$ KRAS exon 2, $3.8 \%$ KRAS exon 3, 6.2\% KRAS exon 4, 2.9\% NRAS exon 2, 4.2\% NRAS exon 3, 0.3\% NRAS exon 4 [21]. In our study, there were 221 cases detected of RAS mutation among 435 valid results $(50.8 \%)$, followed by KRAS exon $2(163,37.5 \%)$, KRAS exon $3(7,1.6 \%)$, KRAS exon $4(6,1.4 \%)$, NRAS exon $2(19,4.3 \%)$, NRAS exon $3(14,3.2 \%)$ and NRAS exon $4(12,2.7 \%)$.

The presence of KRAS mutation indicated an increasing risk of recurrence and death in RASCAL I and RASCAL II study of patients with Duke's stage C disease [22, 23]. In fact, the prognostic impact of KRAS mutation on survival of colorectal cancer patients among advanced disease population was controversial, because many non-EGFR (Epidermal Growth Factor Receptor) containing regimen of systemic treatment failed in exhibiting a difference of outcome between wtKRAS and mtKRAS CRC in some studies [24-27]. On the other hand, RAS mutation may present a negative factor for the surgery treatment of advanced patient $[28,29]$. Kodaz et al. [30] reported that KRAS mutation had a worse prognostic impact on the metastatic CRC patients who underwent curative resection of liver metastasis. Vauthey et al. [31] found out that RAS mutations predicted early lung recurrence among those who had curative resection of colorectal liver metastases and suggested that surgical treatment contributed to prolongation of survival in the population of stage IV colorectal cancer patients and the outcome could be affected by the RAS mutation. In fact, RAS mutation arose early in the process of CRC by polyp-adenoma-neoplasia sequence. A more recent study deduced by Galanopoulos $\mathrm{M}$ and his colleagues and revealed that patients with CRC, polyps and healthy individuals could be discriminated by blood circulation free DNA when they underwent screening colonoscopy, because of the mutations in single nucleotide polymorphism (SNP) of KRAS gene [32]. Currently, preclinical studies have already demonstrated that mtRAS is the potential reason of EGFR target therapy resistance. Results from our study have also suggested that mCRC patients harboring mtRAS molecular characteristics may be a class of population who cannot benefit from active surgery treatment.

Some retrospective studies suggested a trend of survival advantage of PTR. Clancy C et al. [33] deduced a meta-analysis of 21 studies and revealed that PTR for mCRC patients was associated with a lower mortality risk (OR 0.28; $95 \%$ CI 0.165-0.474; P < 0.001) and translated into a difference mean survival of 6.4 months in favor of resection (95 \% CI 5.025- 
7.858, $\mathrm{P}<0.001$ ). Ahmed S et al. [34] found that median survival was 15.2 months (range: 10-30.7 months) in the resection group and 11.4 months (range: 3-22 months) in the nonresection group among mCRC patients from 15 retrospective observational studies. Given that inevitable basic flaws lied in selection bias of these studies, such as good performance status, younger age, oligo metastasis and so on, which suggested that those who experienced PTR could be a better prognosis population among advanced mCRCs patients, the systemic therapy was taken as an canonical initial treatment for the advanced unresectable diseases by the major clinical guidelines from the National Comprehensive Cancer Network (NCCN), the European Society for Medical Oncology (ESMO) and the American Society for Medical Oncology (ASCRS). The non-radical surgery of the primary tumor was not recommended by current clinical guidelines, even if the patients had a good performance and presented no severe tumour related symptoms. Although we compared the prognosis survival of PRT to IPT (Fig. 2, Fig. 3, Table 4), this was not the main research conclusion in our study.

In fact, it is difficult to ethically initiate a prospective randomized controlled clinical trial for asymptomatic unresectable mCRC patients to verify palliative resection or palliative systemic chemotherapy which is an optimal treatment choice for them.

However, Chinese colorectal cancer patients who are initially diagnosed as metastatic diseases are more willing to have a non-radical resection rather than receive palliative systemic chemotherapy until disease progression, which gave us a favorable opportunity to launch this retrospective observational cohort study [35]. In our center, the decision of receiving PTR treatment was made according to patients' intention, surgeons' assessment of surgery treatment risk as well as some other patients' condition and clinical features, such as abdominal pain, bloating and other mild symptoms which suggested probable aggravation.

Although a Consensus Molecular Subtypes (CMS) Consortium analyzed CRC expression profiling data from multiple studies and classified CRC into four CMS groups with distinguishing features: CMS1 (microsatellite instability immune, 14\%), hyper mutated, microsatellite unstable and strong immune activation; CMS2 (canonical, 37\%), epithelial, marked WNT and MYC signaling activation; CMS3 (metabolic, 13\%), epithelial and evident metabolic dysregulation; and CMS4 (mesenchymal, 23\%), prominent transforming growth factor- $\beta$ activation, stromal invasion and angiogenesis [36], the attempts to find out molecular markers that can predict tumour disease outcomes regardless of treatment therapy (prognostic) or that give information about the effect from a specific treatment (predictive) have had limited advance in mCRC. Due to low mutation rate of BRAF about $5 \%$ to $13 \%$, which appeared $5.9 \%(24 / 401)$ in this study, all RAS gene including KRAS and NRAS examination represents a more valuable tool for predictive and prognostic biomarker than BRAF. Gene mutations provide information beyond that provided by the clinic-pathologic characteristics, such as primary tumour size, site (right colon or left colon), multi-/oligometastasis, CEA and CA-199 level, related to the primary tumour as well as metastases, for example, tumor site was not a significant prognostic factors by a multivariate analysis between PTR and IPT.

In addition, the case enrolled in our study was treated much earlier. Patients with asymptomatic unresectable metastases of CRC mainly received systemic chemotherapy and primary tumor resection in our center at that time. Local treatment of liver metastases was not fully in accordance with the current MDT (multidisciplinary team treatment) strategy. Loco regional therapies of the liver metastasis were not commonly used in this study population. Patients with resectable, potentially resectable, potentially topical treatable metastasis were excluded in this study. However, we believed that these did not affect the basic observation of the relationship between RAS gene and survival outcomes in this study. Patients who received PRT were likely to have a better prognosis inherently. It was the purpose of this retrospective cohort study that if they harbored wtRAS gene, they could further benefit from the PTR. 


\section{Cellular Physiology Cell Physiol Biochem 2018;50:768-782 \begin{tabular}{ll|l} 
and Biochemistry Published onlIne: 12 Uctober 2018 & $\begin{array}{l}\text { (C) } 2018 \text { The Author(s). Published by S. Karger AG, Basel } \\
\text { www.karger.com/cpb }\end{array}$ \\
\hline
\end{tabular}}

Liang et al.: RAS Mutations in the Prognosis of Mcrc Patients

\section{Acknowledgements}

This study was funded by the Natural Science Foundation of Science and Technology Committee of Shanghai (14ZR1406500), and Youth Foundation of Zhongshan Hospital (No. 2016ZSQN10).

\section{Disclosure Statement}

The authors have declared no conflicts of interest.

\section{References}

1 Cook AD, Single R, McCahill LE: Surgical resection of primary tumors in patients who present with stage IV colorectal cancer: an analysis of surveillance, epidemiology, and end results data, 1988 to 2000.Ann Surg Oncol 2005;12:637-645.

2 Edge S, Byrd DR, Compton CC, Fritz AG, Greene FL, Trotti A: AJCC Cancer Staging. Springer-Verlag, New York, 2010.

3 Compton CC, Greene FL: The staging of colorectal cancer: 2004 and beyond. CA Cancer J Clin 2004;54:295-308.

4 Makela J, Haukipuro K, Laitinen S, Kairaluoma MI: Palliative operations for colorectal cancer. Dis Colon Rectum 1990;33:846-850.

5 Kim MS, Chung M, Ahn JB, Kim CW, Cho MS, Shin SJ, Baek SJ, Hur H, Min BS, Baik SH, Kim NK: Clinical significance of primary tumor resection in colorectal cancer patients with synchronous unresectable metastasis. J Surg Oncol 2014;110:214-221.

-6 Ahmed S, Fields A, Pahwa P, Chandra-Kanthan S, Zaidi A, Le D, Haider K, Reeder B, Leis A: Surgical Resection of Primary Tumor in Asymptomatic or Minimally Symptomatic Patients With Stage IV Colorectal Cancer: A Canadian Province Experience. Clin Colorectal Cancer 2015;14:e41-47.

7 Tsang WY, Ziogas A, Lin BS, Seery TE, Karnes W, Stamos MJ, Zell JA: Role of primary tumor resection among chemotherapy-treated patients with synchronous stage IV colorectal cancer: a survival analysis. J Gastrointest Surg 2014;18:592-598.

-8 't Lam-Boer J, Mol L, Verhoef C, de Haan AF, Yilmaz M, Punt CJ, de Wilt JH, Koopman M: The CAIR04 study: the role of surgery of the primary tumour with few or absent symptoms in patients with synchronous unresectable metastases of colorectal cancer--a randomized phase III study of the Dutch Colorectal Cancer Group (DCCG). BMC Cancer 2014;14:741.

-9 Rahbari NN, Lordick F, Fink C, Bork U, Stange A, Jager D, Luntz SP, Englert S, Rossion I, Koch M, Buchler MW, Kieser M, Weitz J: Resection of the primary tumour versus no resection prior to systemic therapy in patients with colon cancer and synchronous unresectable metastases (UICC stage IV): SYNCHRONOUS--a randomised controlled multicentre trial (ISRCTN30964555). BMC Cancer 2012;12:142.

$\checkmark 10$ Kim CW, Baek JH, Choi GS, Yu CS, Kang SB, Park WC, Lee BH, Kim HR, Oh JH, Kim JH, Jeong SY, Ahn JB, Baik SH: The role of primary tumor resection in colorectal cancer patients with asymptomatic, synchronous unresectable metastasis: Study protocol for a randomized controlled trial. Trials 2016;17:34.

11 Dorajoo SR, Tan WJ, Koo SX, Tan WS, Chew MH, Tang CL, Wee HL, Yap CW: A scoring model for predicting survival following primary tumour resection in stage IV colorectal cancer patients with unresectable metastasis. Int J Colorectal Dis 2016;31:235-245.

12 de Mestier L, Manceau G, Neuzillet C, Bachet JB, Spano JP, Kianmanesh R, Vaillant JC, Bouche O, Hannoun L, Karoui M: Primary tumor resection in colorectal cancer with unresectable synchronous metastases: A review. World J Gastrointest Oncol 2014;6:156-169.

13 Turner N, Tran B, Tran PV, Sinnathamby M, Wong HL, Jones I, Croxford M, Desai J, Tie J, Field KM, Kosmider S, Bae S, Gibbs P: Primary Tumor Resection in Patients With Metastatic Colorectal Cancer Is Associated With Reversal of Systemic Inflammation and Improved Survival. Clin Colorectal Cancer 2015;14:185-191. 


\section{Cellular Physiology Cell Physiol Biochem 2018;50:768-782 \begin{tabular}{l|l|l} 
and Biochemistry Published onlIne: 12 October 2018 & $\begin{array}{l}\text { C 2018 The Author(s). Published by S. Karger AG, Basel } \\
\text { www.karger.com/cpb }\end{array}$ \\
\hline
\end{tabular}}

Liang et al.: RAS Mutations in the Prognosis of Mcrc Patients

14 Tol J, Nagtegaal ID, Punt CJ: BRAF mutation in metastatic colorectal cancer. N Engl J Med 2009;361:98-99.

15 Douillard JY, Oliner KS, Siena S, Tabernero J, Burkes R, Barugel M, Humblet Y, Bodoky G, Cunningham D, Jassem J, Rivera F, Kocakova I, Ruff P, Blasinska-Morawiec M, Smakal M, Canon JL, Rother M, Williams R, Rong A, Wiezorek J, Sidhu R, Patterson SD: Panitumumab-FOLFOX4 treatment and RAS mutations in colorectal cancer. N Engl J Med 2013;369:1023-1034.

-16 Arrington AK, Heinrich EL, Lee W, Duldulao M, Patel S, Sanchez J, Garcia-Aguilar J, Kim J: Prognostic and predictive roles of KRAS mutation in colorectal cancer. Int J Mol Sci 2012;13:12153-12168.

17 Osumi H, Shinozaki E, Suenaga M, Matsusaka S, Konishi T, Akiyoshi T, Fujimoto Y, Nagayama S, Fukunaga Y, Ueno M, Mise Y, Ishizawa T, Inoue Y, Takahashi Y, Saiura A, Uehara H, Mun M, Okumura S, Mizunuma N, Miki Y, Yamaguchi T: RAS mutation is a prognostic biomarker in colorectal cancer patients with metastasectomy. Int J Cancer 2016;139:803-811.

18 Waring P, Tie J, Maru D, Karapetis CS: RAS Mutations as Predictive Biomarkers in Clinical Management of Metastatic Colorectal Cancer. Clin Colorectal Cancer 2016;15:95-103.

19 Payandeh M, Shazad B, Sadeghi M, Shahbazi M: Correlation between RAS Test Results and Prognosis of Metastatic Colorectal Cancer Patients: a Report from Western Iran. Asian Pac J Cancer Prev 2016;17:1729-1732.

20 Therasse P, Arbuck SG, Eisenhauer EA, Wanders J, Kaplan RS, Rubinstein L, Verweij J, Van Glabbeke M, van Oosterom AT, Christian MC, Gwyther SG: New guidelines to evaluate the response to treatment in solid tumors. European Organization for Research and Treatment of Cancer, National Cancer Institute of the United States, National Cancer Institute of Canada. J Natl Cancer Inst 2000;92:205-216.

21 Peeters M, Kafatos G, Taylor A, Gastanaga VM, Oliner KS, Hechmati G, Terwey JH, van Krieken JH: Prevalence of RAS mutations and individual variation patterns among patients with metastatic colorectal cancer: A pooled analysis of randomised controlled trials. Eur J Cancer 2015;51:1704-1713.

22 Andreyev HJ, Norman AR, Cunningham D, Oates JR, Clarke PA: Kirsten ras mutations in patients with colorectal cancer: the multicenter "RASCAL" study. J Natl Cancer Inst 1998;90:675-684.

23 Andreyev HJ, Norman AR, Cunningham D, Oates J, Dix BR, Iacopetta BJ, Young J, Walsh T, Ward R, Hawkins N, Beranek M, Jandik P, Benamouzig R, Jullian E, Laurent-Puig P, Olschwang S, Muller O, Hoffmann I, Rabes HM, Zietz C et al.: Kirsten ras mutations in patients with colorectal cancer: the 'RASCAL II' study. Br J Cancer 2001;85:692-696.

24 Tol J, Koopman M, Cats A, Rodenburg CJ, Creemers GJ, Schrama JG, Erdkamp FL, Vos AH, van Groeningen CJ, Sinnige HA, Richel DJ, Voest EE, Dijkstra JR, Vink-Borger ME, Antonini NF, Mol L, van Krieken JH, Dalesio O, Punt CJ: Chemotherapy, bevacizumab, and cetuximab in metastatic colorectal cancer. N Engl J Med 2009;360:563-572.

25 Price TJ, Hardingham JE, Lee CK, Weickhardt A, Townsend AR, Wrin JW, Chua A, Shivasami A, Cummins MM, Murone C, Tebbutt NC: Impact of KRAS and BRAF Gene Mutation Status on Outcomes From the Phase III AGITG MAX Trial of Capecitabine Alone or in Combination With Bevacizumab and Mitomycin in Advanced Colorectal Cancer. J Clin Oncol 2011;29:2675-2682.

-26 Hecht JR, Mitchell E, Chidiac T, Scroggin C, Hagenstad C, Spigel D, Marshall J, Cohn A, McCollum D, Stella P, Deeter R, Shahin S, Amado RG: A randomized phase IIIB trial of chemotherapy, bevacizumab, and panitumumab compared with chemotherapy and bevacizumab alone for metastatic colorectal cancer. J Clin Oncol 2009;27:672-680.

-27 Zhou M, Yu P, Qu J, Chen Y, Zhou Y, Fu L, Zhang J: Efficacy of Bevacizumab in the First-Line Treatment of Patients with RAS Mutations Metastatic Colorectal Cancer: a Systematic Review and Network MetaAnalysis. Cell Physiol Biochem 2016;40:361-369.

-28 Karagkounis G, Torbenson MS, Daniel HD, Azad NS, Diaz LA, Jr, Donehower RC, Hirose K, Ahuja N, Pawlik TM, Choti MA: Incidence and prognostic impact of KRAS and BRAF mutation in patients undergoing liver surgery for colorectal metastases. Cancer 2013;119:4137-4144.

29 Renaud S, Romain B, Falcoz PE, Olland A, Santelmo N, Brigand C, Rohr S, Guenot D, Massard G: KRAS and BRAF mutations are prognostic biomarkers in patients undergoing lung metastasectomy of colorectal cancer. Br J Cancer 2015;112:720-728.

-30 Kodaz H, Erdogan B, Hacibekiroglu I, Turkmen E, Tozkir H, Albayrak D, Uzunoglu S, Cicin I: Primary Tumor Resection Offers Higher Survival Advantage in KRAS Mutant Metastatic Colorectal Cancer Patients. Hepatogastroenterology 2015;62:876-879. 


\section{Cellular Physiology Cell Physiol Biochem 2018;50:768-782 and Biochemistry \begin{tabular}{l|l} 
DOI: 10.1159/000494242 & $\begin{array}{l}\text { O 2018 The Author(s). Published by S. Karger AG, Basel } \\
\text { www.karger.com/cpb }\end{array}$
\end{tabular}

Liang et al.: RAS Mutations in the Prognosis of Mcrc Patients

31 Vauthey JN, Zimmitti G, Kopetz SE, Shindoh J, Chen SS, Andreou A, Curley SA, Aloia TA,

Maru DM: RAS mutation status predicts survival and patterns of recurrence in patients undergoing hepatectomy for colorectal liver metastases. Ann Surg 2013;258:619-627.

-32 Galanopoulos M, Papanikolaou IS, Zografos E, Viazis N, Papatheodoridis G, Karamanolis D, Marinos E, Mantzaris GJ, Gazouli M: Comparative Study of Mutations in Single Nucleotide Polymorphism Loci of KRAS and BRAF Genes in Patients Who Underwent Screening Colonoscopy, With and Without Premalignant Intestinal Polyps. Anticancer Res 2017;37:651-657.

-33 Clancy C, Burke JP, Barry M, Kalady MF, Calvin Coffey J: A meta-analysis to determine the effect of primary tumor resection for stage IV colorectal cancer with unresectable metastases on patient survival. Ann Surg Oncol 2014;21:3900-3908.

-34 Ahmed S, Shahid RK, Leis A, Haider K, Kanthan S, Reeder B, Pahwa P: Should noncurative resection of the primary tumour be performed in patients with stage iv colorectal cancer? A systematic review and meta-analysis. Curr Oncol 2013;20:420-441.

35 Wang Z, Liang L, Yu Y, Wang Y, Zhuang R, Chen Y, Cui Y, Zhou Y, Liu T: Primary Tumour Resection Could Improve the Survival of Unresectable Metastatic Colorectal Cancer Patients Receiving Bevacizumab-Containing Chemotherapy. Cell Physiol Biochem 2016;39:1239-1246.

-36 Guinney J, Dienstmann R, Wang X, de Reynies A, Schlicker A, Soneson C, Marisa L, Roepman P, Nyamundanda G, Angelino P, Bot BM, Morris JS, Simon IM, Gerster S, Fessler E, De Sousa EMF, Missiaglia E, Ramay H, Barras D, Homicsko K et al.: The consensus molecular subtypes of colorectal cancer. Nat Med 2015;21:1350-1356. 